\title{
Association between insulin dosage and insulin usage time, and coronary artery lesions in patients with type 2 diabetes and coronary heart disease
}

\author{
XIAO-LI LUO ${ }^{1 *}$, QING-KAI YAN $^{1 *}$, YAN WANG $^{2}$, HUI FANG $^{1}$, \\ HONG-YONG WANG $^{1}$, YUN BAI ${ }^{2}$, CHUN-YU ZENG ${ }^{1}$ and XU-KAI WANG ${ }^{1}$
}

${ }^{1}$ Department of Cardiology, Daping Hospital, The Third Military Medical University, Chongqing 400042; ${ }^{2}$ Department of
Medical Genetics, College of Basic Medical Science, The Third Military Medical University, Chongqing 400038, P.R. China

Received October 15, 2015; Accepted February 29, 2016

DOI: $10.3892 / \mathrm{etm} .2016 .3117$

\begin{abstract}
Insulin is used in the treatment of type 2 diabetes, with its usage reaching $30-50 \%$ in Western countries. The aim of the present study was to determine the association between insulin dosage (ID)/insulin usage time (IT) and coronary artery lesions in patients of type 2 diabetes with coronary heart disease. Based on the insulin using dosage, 353 type 2 diabetes patients were divided into the high-dose $(\geq 0.5 \mathrm{IU} / \mathrm{kg})$ and low-dose $(<0.5 \mathrm{IU} / \mathrm{kg})$ group. Selected coronary angiography was performed and the Gensini score was used to determine the degree of the coronary artery lesions. The homeostasis model assessment-insulin sensitivity (HOMA-IS) index was assessed by HOMA2. Data including age, gender, smoking, body mass index (BMI), systolic blood pressure (SBP), diastolic blood pressure (DBP), low-density lipoprotein cholesterol (LDL-C), high-density lipoprotein cholesterol (HDL-C), total cholesterol (TC), triglyceride (TG), glucose (Glu), hemoglobin A1c (HbA1c), C-peptide, ID, IT, diabetes duration time (DT), and IT/DT were collected. The association between insulin usage (both dose and time) and the coronary artery lesions in these patients was then determined. Statistical differences for the two groups for factors including C-peptide, HbA1c, ID, IT, DT, IT/DT and the Gensini score values $(\mathrm{P}<0.05)$ were identified. By contrast, no significant differences for factors such as gender, smoking history, age, BMI, TC, TG, LDL, HDL, fasting insulin, Glu, SBP and DBP were observed. The coronary artery damage Gensini score in insulin-insensitive individuals was significantly greater than
\end{abstract}

Correspondence to: Dr Xu-Kai Wang, Department of Cardiology, Daping Hospital, Third Military Medical University, Daping Avenue No. 10, Yuzhong District, Chongqing 400042, P.R. China

E-mail:wangxuk@163.com

${ }^{*}$ Contributed equally

Key words: coronary heart disease, diabetes, insulin, coronary angiography, dose that in the insulin-sensitive individuals. The Spearman analysis revealed that ID and IT, DT and IT/DT were positively correlated with the coronary artery damage Gensini score. The multivariate regression, the interquartile range method and receiver operating characteristic analyses showed that ID, ID/DT, IT had a greater effect on coronary vascular damage compared with DT. In conclusion, the degree of coronary artery lesions were correlated with ID, IT, DT, IT/DT. High doses of insulin or a high IT/DT ratio may aggravate coronary artery damage.

\section{Introduction}

Insulin is increasingly used in the treatment of type 2 diabetes (T2D), with its usage reaching 30-50\% in Western countries (1). Nevertheless, many patients receiving insulin therapy develop vascular diseases, such as coronary heart disease and stroke (2). Therefore, the role of insulin in the development of atherosclerosis (AS) is controversial. Previous findings have shown that increased physiological doses of insulin promote vascular endothelial injury, as well as vascular smooth muscle cell proliferation and migration, thereby contributing to the process of AS (3-5). By contrast, it has been reported that insulin delays the process of AS by upregulating nitric oxide synthetase which enhances vasorelaxant function of vascular endothelial cells (6). However, previous studies have not focused on the association between insulin dosage (ID) and usage time, and the coronary artery lesions in patients of T2D and coronary heart disease.

The aim of this study was to examine the association between insulin usage (both dose and time) and coronary artery lesions in these patients. Thhe results showed that high doses of insulin or a high IT/DT ratio may aggravate coronary artery damage.

\section{Materials and methods}

Patients. A total of 353 patients with T2D were recruited in the Department of Cardiology, Daping Hospital, The Third Military School (Chonqing, China). All the patients signed an informed consent prior to participating in the present 
study. The study protocol was approved by the Human Ethics Committee of Daping Hospital.

The inclusion criteria for the present study included, 35-85 years of age and a previous history of T2D, or patients meeting the diagnostic criteria of the '2013 Guidelines for the Prevention and Treatment of Diabetes in China' (7), as well as having undergone coronary arteriography, and ongoing insulin therapy. Exclusion criteria for the study were liver or, kidney disease, anemia [defined as hemoglobin $(\mathrm{Hb})$ of $<60 \mathrm{~g} / \mathrm{l}$ ], tumor or severe malnutrition. Patients were also excluded if they were using glucagon-like peptide-1 receptor agonists, rosiglitazone, dipeptidyl peptidase- 4 inhibitors, or $\alpha$-glucosidase inhibitors within 3 months of screening.

The patients were divided into the high-dose $(\geq 0.5 \mathrm{IU} / \mathrm{kg})$ and low-dose $(<0.5 \mathrm{IU} / \mathrm{kg})$ groups based on insulin usage dosage (8).

Patient information. Patient information such as age, gender, smoking, measured height, weight, systolic blood pressure (SBP), diastolic blood pressure (DBP), ID, insulin usage time (IT), diabetes duration time (DT), caculated body mass index (BMI) and the IT/DT ratio were collected.

Biochemical analysis. After $12 \mathrm{~h}$ of fasting, $3 \mathrm{ml}$ of fasting venous blood was collected in the morning of the second day after hospitalization. Low-density lipoproteincholesterol (LDL-C), high-density lipoprotein cholesterol (HDL-C), total cholesterol (TC), triglycerides (TG), fasting blood glucose (Glu), HbA1c, and C-peptide were quantified using automatic biochemical analyzers (Model Beckman DxC800; Beckman Coulter, CA, USA) and the Cobas 8000 (Model Roche Diagnostics, Roche, IN, USA).

Calculation of Gensini score. The Gensini score was calculated by assigning a severity score on each coronary stenosis, based on the degree of luminal narrowing and geographic importance. Reduction in the lumen diameter, and the radiologic appearance of concentric lesions and eccentric plaques were assessed. Reductions of 25, 50, 75, 90 and 99\%, and complete occlusion were allocated a Gensini score of $1,2,4,8,16$ and 32. Involvement of each principal vascular segment was assigned a multiplier defined by the functional significance of the myocardial area supplied by that segment. Thus, the left main coronary artery received a multiplier $\mathrm{x} 5$, proximal segment of the left anterior descending coronary artery $-x 2.5$, proximal segment of the circum flex artery $-\mathrm{x} 2.5$, mid-segment of the left anterior descending coronary artery -x1.5, while right coronary artery, distal segment of the left anterior descending coronary artery, posterolateral artery, and obtuse marginal artery received a multiplier $\mathrm{x} 1$. The sum of the adjusted results made the total Gensini score.

Statistical analysis. Data were analyzed using SPSS 18.0 software (SPSS, Chongqing, China). Data were presented as the mean \pm standard deviation. Due to large individual differences of fasting insulin (FINS) levels and coronary Gensini scores, the data were analyzed following a logarithm transition.

A one-way analysis of variance was utilized when the variance was homogeneity. A non-parametric test was used for parameters when the variance was heteroscedasticity.
Table I. Comparison of patients' demographic and biochemical characteristics between low- and high-dose insulin groups.

\begin{tabular}{|c|c|c|c|}
\hline Parameters & $\begin{array}{l}\text { Low-dose } \\
\text { insulin }\end{array}$ & $\begin{array}{l}\text { High-dose } \\
\text { insulin }\end{array}$ & $\mathrm{P}$-value \\
\hline Gender (male/female) & $179(105 / 74)$ & $174(92 / 82)$ & 0.099 \\
\hline Age, years & $64.91 \pm 9.59$ & $66.62 \pm 8.85$ & NS \\
\hline Smoking history & 76 & 84 & NS \\
\hline BMI, $\mathrm{kg} / \mathrm{m}^{2}$ & $25.34 \pm 3.22$ & $25.08 \pm 3.21$ & NS \\
\hline LDL-C, mmol/1 & $2.46 \pm 0.79$ & $2.69 \pm 0.78$ & 0.063 \\
\hline HDL-C, mmol/l & $1.10 \pm 0.30$ & $1.04 \pm 0.31$ & NS \\
\hline $\mathrm{TC}, \mathrm{mmol} / \mathrm{l}$ & $4.43 \pm 1.13$ & $4.75 \pm 1.26$ & NS \\
\hline $\mathrm{TG}, \mathrm{mmol} / \mathrm{l}$ & $1.79 \pm 1.36$ & $1.92 \pm 1.48$ & NS \\
\hline Insulin, pmol/l & $1.91 \pm 0.55$ & $1.88 \pm 0.50$ & NS \\
\hline Glu, mmol/1 & $8.54 \pm 3.91$ & $9.12 \pm 3.36$ & NS \\
\hline $\mathrm{HbA1c}, \mathrm{mmol} / \mathrm{l}$ & $7.92 \pm 1.97$ & $8.75 \pm 2.09$ & 0.013 \\
\hline C-peptide, mmol/l & $2.86 \pm 1.90$ & $2.27 \pm 1.75$ & 0.048 \\
\hline $\mathrm{SBP}, \mathrm{mmHg}$ & $146.52 \pm 25.83$ & $152.15 \pm 28.48$ & NS \\
\hline $\mathrm{DBP}, \mathrm{mmHg}$ & $82.30 \pm 14.35$ & $81.92 \pm 13.74$ & NS \\
\hline Insulin dose, IU & $20.37 \pm 7.08$ & $38.74 \pm 8.98$ & $<0.001$ \\
\hline DT, years & $8.24 \pm 5.05$ & $13.27 \pm 8.19$ & $<0.001$ \\
\hline IT, years & $3.05 \pm 2.62$ & $6.50 \pm 5.42$ & $<0.001$ \\
\hline Ratio of IT/DT & $0.41 \pm 0.27$ & $0.49 \pm 0.19$ & 0.034 \\
\hline Gensini score & $30.82 \pm 30.07$ & $48.20 \pm 36.76$ & 0.002 \\
\hline
\end{tabular}

BMI, body mass index; LDL-C, low-density lipoprotein cholesterol; HDL-C, high-density lipoprotein cholesterol; TC, total cholesterol; TG, triglyceride; Glu, glucose; HbA1c, hemoglobin A1c; SBP, systolic blood pressure; DBP, diastolic blood pressure; DT, diabetes duration time; IT, insulin usage time; NS, not significant. Data are presented as absolute numbers or as the mean \pm standard deviation.

The homeostasis model assessment-insulin sensitivity (HOMA-IS) index was assessed by HOMA2. Based on the HOMA-insulin resistance (IR) $(50 \%)$, the patients were classified into insulin-sensitive and -insensitive individuals. Analysis between ID, IT, DT, and IT/DT correlations with the coronary artery damage Gensini score was determined, where the correlative degree was expressed by the correlation coefficient (r). Multivariate regression analysis among ID, IT, DT, and IT/DT was performed to determine the correlations with the coronary artery damage Gensini score. ID, IT, DT, and IT/DT were compared between the subgroups, according to the interquartile range (IQR) method (Q1, Q2, Q3 and Q4). The ID, IT, DT, and IT/DT values of patients with coronary artery disease (Gensini score $>0$ ) were analyzed with receiver operating characteristic (ROC) curves. $\mathrm{P}<0.05$ was considered to indicate a statistically significant difference.

\section{Results}

Patient data and outcomes were compared between the high- and low-dose groups (Table I). Statistically significant differences were observed between the two groups for the levels of insulin C-peptide, HbA1c, ID, IT, DT, the IT/DT ratio and Gensini's score values $(\mathrm{P}<0.05$, the high- vs. low-dose 
Table II. Insulin-sensitivity vs. coronary artery damage.

\begin{tabular}{lccc}
\hline & $\begin{array}{c}\text { Insulin-insensitive } \\
\text { Parameter }\end{array}$ & $\begin{array}{c}\text { Insulin-sensitive } \\
\text { patients, } \mathrm{n}=180\end{array}$ & P-value \\
\hline Gensini score & $45.08 \pm 37.79$ & $33.89 \pm 30.31$ & 0.044 \\
\hline
\end{tabular}

Data are presented as mean \pm standard deviation.

Table III. Correlation between insulin dose, IT, DT, the IT/DT ratio, and Gensini score.

\begin{tabular}{lcccc}
\hline Parameter & $\begin{array}{c}\text { Insulin } \\
\text { dose }\end{array}$ & IT & DT & $\begin{array}{c}\text { Ratio } \\
\text { of IT/DT }\end{array}$ \\
\hline $\begin{array}{l}\text { Gensini } \\
\text { score } \\
\text { P-value }\end{array}$ & $\mathrm{r}=0.300$ & $\mathrm{r}=0.319$ & $\mathrm{r}=0.163$ & $\mathrm{r}=0.281$ \\
\hline DT, diabetes duration time; IT, insulin usage time. & \\
\hline
\end{tabular}

Table IV. Multivariate regression analysis of association between insulin dose, IT, DT, the IT/DT ratio, and Gensini score.

\begin{tabular}{lcccc}
\hline Parameters & $\begin{array}{c}\text { Insulin } \\
\text { dose }\end{array}$ & IT & DT & $\begin{array}{c}\text { Ratio } \\
\text { of IT/DT }\end{array}$ \\
\hline Odds ratio & 0.230 & 0.142 & 0.214 & 0.300 \\
P-value & 0.007 & 0.410 & 0.166 & 0.009 \\
\hline
\end{tabular}

DT, diabetes duration time; IT, insulin usage time.

groups). No significant differences were observed regarding patients' gender, smoking history, age, BMI, the levels of TC, TG, LDL, HDL, FINS levels, Glu, or SBP or DBP $(\mathrm{P}<0.05)$ (Table I).

Based on the HOMA-IR (50\%), the patients were classified as insulin-sensitive $(n=173)$ and -insensitive $(n=180)$ (Table II). The Gensini score in insulin-insensitive patients was significantly greater than that in insulin-sensitive patients $(\mathrm{P}<0.05)$ (Table II).

In addition, ID, IT, DT, and the IT/DT ratio positively correlated with the Gensini score $(\mathrm{P}<0.05)$ (Table III).

The multivariate regression analysis revealed which factors were associated with changes in ID $(\mathrm{OR}=0.230, \mathrm{P}<0.01)$ and the $\mathrm{IT} / \mathrm{DT}$ ratio $(\mathrm{OR}=0.300, \mathrm{P}<0.01)$ (Table IV).

Based on the IQR method, indices such as ID $(\leq 20,20-30$, $30-40, \geq 40)$, IT ( $\leq 1,1-3,3-6, \geq 6)$, DT $(\leq 5,5-10,10-14, \geq 14)$, and the IT/DT ratio $(\leq 0.3,0.3-0.4,0.4-0.6, \geq 0.6)$, were compared between subgroups (Q1, Q2, Q3 and Q4) (Table V).

Statistics showed that the coronary artery damage Gensini score of the highest indicator subgroup (Q4) was significantly higher than that in the lowest indicator subgroup (Q1). In addition to IT, there were also significant differences between the remainig three indices of the subgroups (Fig. 1).
Table V. Analysis of insulin dose, IT, DT, and the IT/DT ratio in subgroups by the interquartile range method.

\begin{tabular}{lcccc}
\hline Parameters & Q1 & Q2 & Q3 & Q4 \\
\hline Insulin dose & $<20$ & $20-30$ & $30-40$ & $>40$ \\
IT & $\leq 1$ & $1-3$ & $3-6$ & $\geq 6$ \\
DT & $\leq 5$ & $5-10$ & $10-14$ & $\geq 14$ \\
Ratio of IT/DT & $<0.3$ & $0.3-0.4$ & $0.4-0.6$ & $>0.6$ \\
\hline
\end{tabular}

DT, diabetes duration time; IT, insulin usage time.

Table VI. Analysis of insulin dose, IT, DT, and the IT/DT ratio in subgroups by ROC method.

\begin{tabular}{lcccc}
\hline Parameters & Area & P-value & Lower limit & Upper limit \\
\hline Insulin dose & 0.820 & 0.001 & 0.696 & 0.945 \\
IT & 0.708 & 0.028 & 0.552 & 0.864 \\
DT & 0.637 & 0.149 & 0.552 & 0.864 \\
Ratio of IT/DT & 0.733 & 0.014 & 0.599 & 0.867 \\
\hline
\end{tabular}

ROC, receiver operating characteristic; DT, diabetes duration time; IT, insulin usage time.

We carried out a ROC curve analysis on ID, IT, DT, and the IT/DT ratio. ROC analysis revealed that the ID area under the ROC curve was 0.820 (range, 0.696-0.945), the IT/DT area under the ROC curve was 0.733 (range, 0.599-0.867), and IT area under the ROC curve was 0.708 (range, 0.552-0.864). These indices were statistically significant (Table VI). However, the DT ROC curve did not obtain statistical significance (Fig. 2).

\section{Discussion}

At present, insulin is used early to control blood Glu of patients with T2D (9-10). Excessive use of insulin is common in China (11). However, such excessive use of insulin potentially leads to hypoglycemia, insulin resistance and even hyperinsulinemia (2). Thus, the effect insulin has on the occurrence and development of AS remains controversial. It has been shown that high doses of insulin reduce plasma levels of interleukin-6, interleukin-8, and tumor necrosis factor- $\alpha$, thereby attenuating inflammatory processes, promoting heart metabolism, and facilitating the recovery of myocardial cells and its myocardial functions (12-15). However, high doses of FINS (ID) aggravate the severity of coronary artery disease $(3,4)$. In addition, no studies have been conducted thus far with regard to the association between IT, DT and coronary AS. Therefore, IT and DT were included in the present study. We observed a positive association between IT, DT and Gensini scores. In order to show the extent of early treatment of insulin, the concept of 'insulin usage time/diabetes duration time (IT/DT)' was applied in the study, for the first time, to the best of our knowledge. A positive association between IT/DT and Gensini score was also identified. The ROC analysis clearly revealed 
A

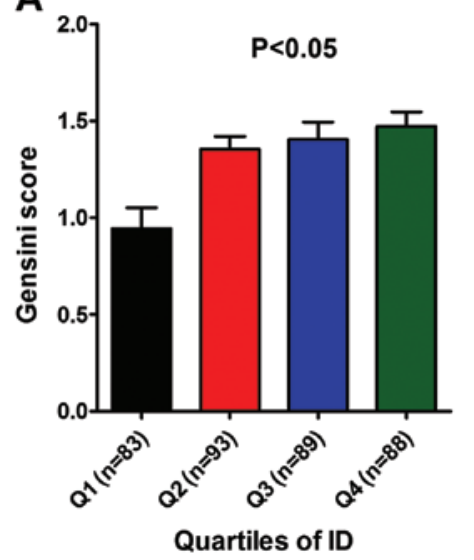

C

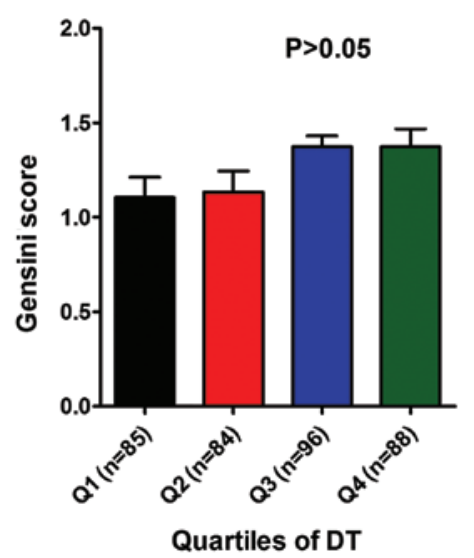

B

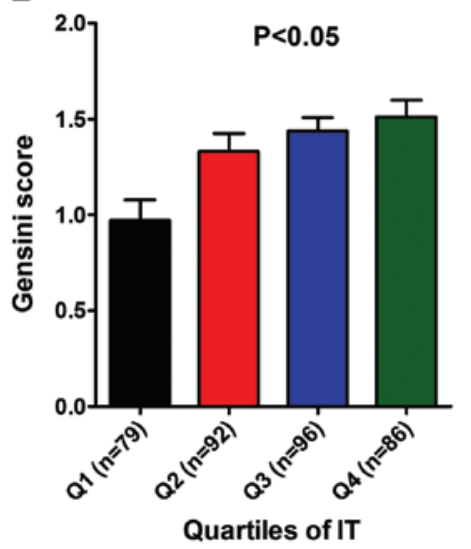

D

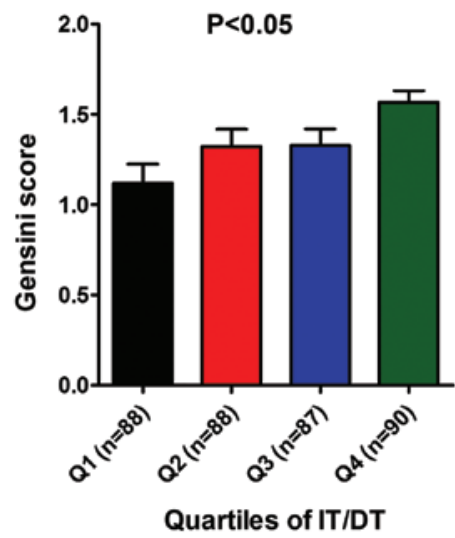

Figure 1. (A) Insulin dosage (ID), (B) insulin usage time (IT), (C) diabetes duration time (DT), and (D) IT/DT indices were classified by subgroups, according to the interquartile range method-based on their levels (Q1, Q2, Q3 and Q4). After the classification, the coronary artery damage Gensini score of each subgroup was compared between the subgroups.

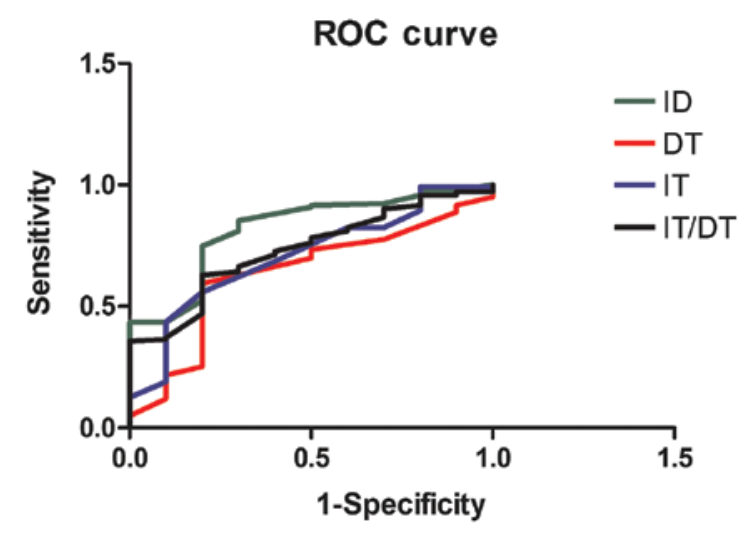

Figure 2. The receiver operating characteristic (ROC) curve analysis between insulin dosage (ID), insulin usage time (IT), diabetes duration time (DT), IT/DT and the coronary artery damage, assessed by Gensini scores.

that ID, IT, IT/DT were predictive of the severity of coronary artery damage

Long-term administration of exogenous insulin can lead to lipid abnormality and vascular wall thickening $(4,16)$. In animals, insulin induces experimental AS (4). Coronary heart disease and AS induced by high-dose insulin may be due to: i) stimulation of proliferation and migration of arterial smooth muscle cells (17), ii) stimulation of uptake of cholesterol and LDL-C by endothelial cells of capillary vessels, which causes a combination of LDLs with arterial smooth muscle cells and mononuclear macrophage (18-21), iii) release of platelet-derived growth factor, insulin-like growth factor-1 and other inflammatory factors, by activating insulin mitogen-activated protein kinase/phosphoinositide 3-kinase signal transduction pathways using high-dose insulin. Combined, this inhibits release of nitric oxide, causing endothelial cell damage and oxidative stress, and leading to AS (22-24). Thus, hyperinsulinemia may be a risk factor of coronary heart disease and AS (25-27). Our finding showed statistical differences between the high-dose group $(\geq 0.5 \mathrm{IU} / \mathrm{kg})$ and low-dose group $(<0.5 \mathrm{IU} / \mathrm{kg})$, such as insulin C-peptide, HbAlc, ID, DT, IT, IT/DT and the Gensini's score values $(\mathrm{P}<0.05)$. The correlation analysis revealed the ID, IT, DT and IT/DT values were positively correlated with the coronary artery damage Gensini score. These data suggest that the degree of the coronary artery lesions were correlated with insulin doses or insulin usage time.

In the present study, insulin-insensitive patients had significantly higher Gensini scores. Based on this, we suggest that for T2D patients who do not take lifestyle intervention on diet, physical activity and smoking-insulin sensitivity should be calculated based on blood Glu and FINS levels when selecting the insulin-sensitizing treatment. If the effect is not 
satisfactory, exogenous insulin therapy should be administered based on weight to avoid overdosing. If recovery of the islet cell sensitivity to Glu, tested by HOMA, in T2D patients after insulin use occurs, the treatment plan should be adjusted to avoid long-term excessive exposure to insulin and minimize the damage of insulin to blood vessels.

In conclusion, the results of the present study suggest that in T2D patients in, higher ID or the ratio IT/DT are associated with more serious coronary injury. This finding suggests that an early and longer-term administration of high-dose of insulin may negatively impact coronary vessels.

\section{Acknowledgements}

The present study was supported by a Chinese National Natural Science Foundation grant to X.K. Wang (grant no. 81170281).

\section{References}

1. 2010 U.S. Type 2 Diabetes Patients' Choice: Awareness, Usage, and Preferences of Insulin Injection Pens. Frost \& Sullivan, 2011.

2. Rensing KL, Reuwer AQ, Arsenault BJ, von der Thüsen JH, Hoekstra JB, Kastelein JJ and Twickler TB: Reducing cardiovascular disease risk in patients with type 2 diabetes and concomitant macrovascular disease: can insulin be too much of a good thing? Diabetes Obes Metab 13: 1073-1087, 2011.

3. Yanase M, Takatsu F, Tagawa T, Kato T, Arai K, Koyasu M, Horibe H, Nomoto S, Takemoto K, Shimizu S and Watarai M: Insulin resistance and fasting hyperinsulinemia are risk factors for new cardiovascular events in patients with prior coronary artery disease and normal glucose tolerance. Circ J 68: 47-52, 2004.

4. Caccamo G, Bonura F, Bonura F, Vitale G, Novo G, Evola S, Evola G, Grisanti MR and Novo S: Insulin resistance and acute coronary syndrome. Atherosclerosis 211: 672-675, 2010.

5. Breen DM and Giacca A: Effects of insulin on the vasculature. Curr Vasc Pharmacol 9: 321-332, 2011.

6. Kuboki K, Jiang ZY, Takahara N, Ha SW, Igarashi M, Yamauchi T, Feener EP, Herbert TP, Rhodes CJ and King GL: Regulation of endothelial constitutive nitric oxide synthase gene expression in endothelial cells and in vivo: a specific vascular action of insulin. Circulation 101: 676-681, 2000.

7. Chinese Diabetes Society (CDS): Chinese guideline for type 2 diabetes prevention (2013). Chin J Diab 22: 2-42, 2014 (In Chinese).

8. Leahy JL and Cefalu WT (eds): Insulin Therapy. Marcel Dekker, Inc., New York, NY, pp193-222, 2002.

9. Permana $\mathrm{H}$ : How to initiate and intensification of insulin therapy in type $2 \mathrm{DM}$ patients with obesity? Obes Res Clin Pract J 7 (Suppl 1): 7-8, 2013.

10. No authors listed: Summary of revisions for the 2009 Clinical Practice Recommendations. Diabetes Care 32 (Suppl 1): S3-S5, 2009.

11. Endocrinology society of Chinese Medical Association: Chinese expert consensus on the clinical application of adult type 2 diabetes insulin. Chin J Endocrinol Metab 29: 1-6, 2013.

12. Albacker T, Carvalho G, Schricker T and Lachapelle $K$ : High-dose insulin therapy attenuates systemic inflammatory response in coronary artery bypass grafting patients. Ann Thorac Surg 86: 20-27, 2008.
13. Owens DR: Stepwise intensification of insulin therapy in type 2 diabetes management - exploring the concept of the basal-plus approach in clinical practice. Diabet Med 30: 276-288, 2013.

14. Albacker TB, Carvalho G, Schricker T and Lachapelle K: Myocardial protection during elective coronary artery bypass grafting using high-dose insulin therapy. Ann Thorac Surg 84: 1920-1927, 2007.

15. Mahajan R, Daga MK and Bhattacharjee J: Hyperinsulinemia in subjects with and without coronary artery disease: a preliminary study from North India. Indian Heart J 54: 687-691, 2002.

16. Chistiakov DA, Sobenin IA, Orekhov AN and Bobryshev YV: Mechanisms of medial arterial calcification in diabetes. Curr Pharm Des 20: 5870-5883, 2014

17. Siddals KW, Allen J, Sinha S, Canfield AE, Kalra PA and Gibson JM: Apposite insulin-like growth factor (IGF) receptor glycosylation is critical to the maintenance of vascular smooth muscle phenotype in the presence of factors promoting osteogenic differentiation and mineralization. J Biol Chem 286: 16623-16630, 2011.

18. Kim JA, Montagnani M, Koh KK and Quon MJ: Reciprocal relationships between insulin resistance and endothelial dysfunction: molecular and pathophysiological mechanisms. Circulation 113: 1888-1904, 2006

19. Purohit P: A correlation study of CVD risk factors in Type 2 diabetics of Western Rajasthan. Int J Diabetes Dev Ctries: 190-197, 2014.

20. Adiels M, Borén J, Caslake MJ, Stewart P, Soro A, Westerbacka J, Wennberg B, Olofsson SO, Packard C and Taskinen MR: Overproduction of VLDL1 driven by hyperglycemia is a dominant feature of diabetic dyslipidemia. Arterioscler Thromb Vasc Biol 25: 1697-1703, 2005.

21. Tangvarasittichai S, Poonsub $\mathrm{P}$ and Tangvarasittichai $\mathrm{O}$ : Association of serum lipoprotein ratios with insulin resistance in type 2 diabetes mellitus. Indian J Med Res 131: 641-648, 2010.

22. Hill MM, Connolly LM, Simpson RJ and James DE: Differential protein phosphorylation in 3T3-L1 adipocytes in response to insulin versus platelet-derived growth factor. No evidence for a phosphatidylinositide 3-kinase-independent pathway in insulin signaling. J Biol Chem 275: 24313-24320, 2000.

23. Abhijit S, Bhaskaran R, Narayanasamy A, Chakroborty A, Manickam N, Dixit M, Mohan V and Balasubramanyam M: Hyperinsulinemia-induced vascular smooth muscle cell (VSMC) migration and proliferation is mediated by converging mechanisms of mitochondrial dysfunction and oxidative stress. Mol Cell Biochem 373: 95-105, 2013.

24. Cohen P: The twentieth century struggle to decipher insulin signalling. Nat Rev Mol Cell Biol 7: 867-873, 2006.

25. Ning J and Clemmons DR: AMP-activated protein kinase inhibits IGF-I signaling and protein synthesis in vascular smooth muscle cells via stimulation of insulin receptor substrate 1 S794 and tuberous sclerosis 2 S1345 phosphorylation. Mol Endocrinol 24: 1218-1229, 2010.

26. Isenović ER, Kedees MH, Tepavcević S, Milosavljević T, Korićanac G, Trpković A and Marche P: Role of PI3K/AKT, cPLA2 and ERK1/2 signaling pathways in insulin regulation of vascular smooth muscle cells proliferation. Cardiovasc Hematol Disord Drug Targets 9: 172-180, 2009.

27. Doronzo G, Viretto M, Russo I, et al: Insulin increases matrix metalloproteinase-2 synthesis, secretion and activity in aortic vascular smooth muscle cells through the PI3-K pathway: effect reduced by insulin resistance. Diabetologia 51: S253-S254, 2008. 